\title{
Statin-induced myopathy - a challenge for patients and physicians
}

\author{
Gabriela Anca ANGELESCU ${ }^{1,2}$, Mirela Marioara TOMA ${ }^{3}$, Valentin VARLAS ${ }^{2,4}$ \\ ${ }^{1}$ Department of Internal Medicine, Ilfov County Clinical Hospital, Bucharest, Romania \\ 2"Carol Davila" University of Medicine and Pharmacy, Bucharest, Romania \\ ${ }^{3}$ Department of Pharmacy, Doctoral School of Biomedical Sciences, Faculty of Medicine and \\ Pharmacy, University of Oradea, Romania \\ ${ }^{4}$ Department of Obstetrics and Gynaecology, Filantropia Clinical Hospital, Bucharest, Romania
}

\begin{abstract}
Statins are the most frequently used drugs for lowering the low density lipoprotein (LDL)-cholesterol and are proven to decrease the cardiovascular-driven mortality. The statin-induced myopathy represents a side effect of the statin therapy and includes heterogenous muscular symptoms, associated with an increase in the creatine kinase (CK) levels, according to clinical trials, at an incidence of $1.5-5 \%$. The statin-induced myopathy is challenging for clinicians, in order to maintain the specific therapeutic approach for each case, as well as for patients at a high risk of cardiovascular diseases, who acknowledges the long-term benefits of the treatment. The aim of this review article is to showcase the types of statin-induced myopathy, the patient cohorts susceptible of developing this side effect and the treatment strategies for hypercholesterolemial dyslipidemia.
\end{abstract}

Keywords: statin-induced myopathy, creatine kinase, side effect

\section{INTRODUCTION}

Statins represent a class of drugs extremely useful in the prevention of cardiovascular diseases, which account for a yearly death toll of over 4 million in Europe (1). It is demonstrated that low density lipoprotein-cholesterol (LDL-C) is associated with the risk of atherosclerotic cardiovascular disease $(2,3)$.

Statins are highly efficient drugs in decreasing the LDL-C through inhibiting the anti-3-hydroxy-3-methylglutaril-coenzyme A (HMG-CoA) reductase enzyme, which transform HMG-CoA in mevalonate; by blocking the mevalonate pathway, all the intermediates and cholesterol in the hepatocytes are reduced. In response to the decrease the hepatic cholesterol, LDL-receptors present an up-regulation, and subsequently the level of circulant cholesterol is diminished. By inhibiting some intermediates of the mevalonate pathway, for ex- ample ubiquinone (coenzyme Q10), there is a risk to produce muscle injury (4).

Statins are well-tolerated drugs. However, some patients develop muscular side effects, with an incidence of $1.5-3 \%$ reported in randomised trials and $10-13 \%$ in prospective trials, known as the statin-induced myopathy (1). Initially, myalgia, myositis and rhabdomyolysis have been described as potential types of muscular damages. In 2010, the statin-associated autoimmune anti-HMG-CoA reductase antibodies myopathy (anti-HMGCR) was described $(5,6,7)$.

Different criteria for statin classification exist. Depending on solubility, these are: lipophilic (simvastatin, atorvastatin, fluvastatin); hydrophilic (pravastatin, rosuvastatin).

Depending on the intensity of the effect, this is related to dosage. According to American College of Car- 
diology/American Heart Association classification of intensity, a high-intensity reduction on $\mathrm{LDL}-\mathrm{C}>50 \%$ can be obtained only with atorvastatin 40 or $80 \mathrm{mg} /$ day and with rosuvastatin 20 or $40 \mathrm{mg} /$ day, while a moderate-intensity reduction of LDL-C $30-50 \%$ can be reached with all statins (atorvastatin $10-20 \mathrm{mg} /$ day, rosuvastatin 5-10 mg, simvastatin $20-40 \mathrm{mg}$, pravastatin $40-80$ $\mathrm{mg}$, lovastatin $40 \mathrm{mg}$ ). If the aim is to reduce the LDL-C $<30 \%$, then all statins are suitable, but at a low dosage, except for atorvastatin and rosuvastatin, used in the first two situations $(8,9)$.

\section{OBJECTIVE}

The objective of this paper is to review studies and literature about statin-induced myopathy, mechanisms, risk factors, drugs interactions, classification and management in clinical practice, according to the guidelines.

\section{METHODS}

Search review and guidelines by using electronic databases (on PubMed). The research words are "statin", "statin-related myopathy", "creatin kinase level", "coenzyme Q10". 40 articles are cited (between 2002 and 2020).

\section{RESULTS AND DISCUSSION}

From a clinical viewpoint, the symptoms of statin-induced myopathy include muscle pain, muscle weakness, hypersensitivity and nocturnal cramps. The proximal skeletal muscles are affected and the pain is generalised, bilateral and enhanced by physical exercise. The time period between the start of the therapy and the onset of symptoms varies up to 6 months. The symptoms can last $2-4$ months until recovery $(10,11)$. The mechanism of muscle damage by statins is still not clear, but there are three hypotheses in the literature:

1. The decrease of cholesterol level secondary to statin therapy induces alterations in myocytes membrane. Reduced sarcolemmal cholesterol is thought to disturb the myocyte integrity. However, experimental studies do not validate this theory $(12,13)$.

2. The decrease in myocyte apoptosis due to lowered levels of isoprenoids. Isoprenoids are lipids that play a role in cell death, secondary to increased concentration of calcium in cytosol $(5,12)$.

3. The statin-induced coenzyme Q10 inhibition, negatively impacting the mitochondrial energy production, vital for myocytes $(4,14)$. Studies have demonstrated that statins reduce the lev- el of coenzyme Q10 in serum, but not in the myocytes $(14,15)$.

The serum level of creatine kinase (CK) is important to be determined, as the values help clinicians to distinguish between different types of myopathy and to monitor patients with trauma, drug-induced muscle injury and toxicity. CK is an enzyme involved in muscle contraction. Its activity is the greatest in skeletal muscles, heart muscle and brain. Regarding the level of CK, an increase even in the absence of the muscular symptoms could mean other simultaneous neuromuscular disorders: inflammatory myopathies (myositis, dermatomyositis), infectious myopathies, muscular dystrophies, endocrine disease-related myopathies (hypothyroidism) or other previous activities or medical history, such as intense physical exercise, repeated intramuscular injections, recent traumatisms, high fever, shivering, spasms, renal failure or other patients in a critical state $(16,17,18)$.

\section{Description of myopathy subgroups}

Myalgia implies muscle pain, mild or severe, with CK levels normal or increased $<10 x$ upper limit normal (ULN). Normal CK levels and myalgia are reported in $0.3-33 \%$ of cases, in studies, while increased levels of CK $<10$ ULN are reported in 2/1000 patients.

Myositis implies severe muscles symptoms and increase in CK > 10x ULN, but < 50x ULN. In all these patients, renal function must be checked. In literature, the reported incidence is $0.11 \%$ (18).

An increase of $>50 x$ ULN implies rhabdomyolysis and autoimmune myopathies, severe but rare complications (Table 1 ).

There is a fourth situation in patients with no muscular symptoms, but with mild CK elevation < 4x ULN, with an incidence of $1.5-26 \%(5,19,20)$.

Regarding rhabdomyolysis, the muscular pain is severe, with necrosis and myoglobinuria; this could lead to acute renal failure through the precipitates of the myoglobin in renal tubules, and, in rare situations, to death. Rhabdomyolysis occurs in 0.1-4/100,000 patients/year. The level of CK is $>50 x$ ULN. Early diagnosis and treatment bring a favourable outcome. It implies immediate stopping of the statin administration and admission to the hospital to receive treatment with intravenous fluids to prevent renal failure, diuresis and to correct the levels of electrolytes, acid-base and metabolic abnormalities; close monitoring of the patient is required. Dialysis may be necessary in patients with oliguric renal failure. The efficacy of vitamin E, C and pentoxifylline remains unclear, according to controlled studies (21-24).

The other severe type of myopathy is autoimmune-mediated necrotising myositis or the so-called autoimmune anti HMG-CoA reductase antibodies myo- 
TABLE 1. Description of myopathy subgroups

\begin{tabular}{|c|c|}
\hline Myopathy & Description \\
\hline Rhabdomyolysis & $\begin{array}{l}\text { - Symptoms include severe muscle pain, necrosis and myoglobinuria, which could lead to acute renal failure } \\
\text { through the toxicity of myoglobin in the renal tubules or death, } \\
\text { - CK level increases over } 100 x \text { normal } \\
\text { - Implies the immediate, permanent halt of statin administration, hospitalisation, hydration and monitoring } \\
\text { the renal function of the patient. } \\
\text { - Frequency of } 1-3 \text { cases } / 100,000 \text { patients/year }(1,10,28)\end{array}$ \\
\hline $\begin{array}{l}\text { Autoimmune } \\
\text { anti-HMG-CoA } \\
\text { reductase (anti- } \\
\text { HMGCR) myopathy }\end{array}$ & $\begin{array}{l}\text { - First described in } 2010 \\
\text { - Following the halt of statin administration, the muscular symptoms and increased CK level persisted. } \\
\text { inflammation. } \\
\text { - Rare, } 2-3 \text { cases } / 100,000 \text { treated patients. } \\
\text { - HLA DRB1 gene-associated. } \\
\text { - Treatment implies administering high doses of cortisone, immunosuppressors, immunoglobulin IV and } \\
\text { monoclonal antibodies (Rituximab). } \\
\text { - Re-administration of statin can lead to the return of disease }(6,19) \text {. }\end{array}$ \\
\hline
\end{tabular}

pathy (anti HMGCR). It was first described in 2010 and currently it must be suspected in those patients with muscular symptoms and CK elevation, despite statin cessation. The frequency is 2 cases/ 1 million patients/ year and it is increased with age. The diagnosis implies the assessment of the anti HMGCR antibodies and muscular biopsy, revealing necrosis and inflammation. Antibodies occur in $94 \%$ of affected individuals. It is associated with genetic factors such as HLA-DR11 and HLA-DRB1 allele. The levels of CK are very high (10-100x ULN) in $90 \%$ of cases. Electromyography could be necessary. The treatment implies stopping the statin and initiation of immunosuppressive medication: corticosteroids, for example, prednisone $1 \mathrm{mg} / \mathrm{kg}$ body weight, methotrexate, azathioprine or mycophenolate. If the therapy fails, immunoglobulin can be used intravenously (particularly in patients with diabetes mellitus) or rituximab $(6,13,19,22,24-27)$.

Establishing the risk of myopathy in patients is highly critical before initiating statin therapy and is directly linked to both clinical and paraclinical patient monitoring (Table 2). Through the analysis of statin-induced myopathy-developing patients, risk factors for its debut have been identified and have been split in drug-related and patient-related risks.
The evaluation of the ongoing statin treatment scheme, as part of the analysis of the muscular symptoms carefully conducted by the physician, should be included. Statins are metabolised through the cytochrome CYP450 pathway and other drugs that normally inhibit this substrate and are being administered could increase the risk of statin toxicity. The debut of statin-induced myopathy is favoured by the simultaneous administration of fluconazole, amiodarone, ciclosporin, fibrates $\mathrm{H} 2$ receptor blockers, macrolides, non-hydropyridine calcium channel antagonists, protease inhibitors and warfarin $(1,30,31)$.

Another driver for myopathy is the genetic predisposition, particularly the SLCO1B1 (Solute Carrier Organic Anion Transporter Family Member 1B1) gene polymorphism associated with a high risk of developing myopathy in patients on simvastatin therapy. There is currently no knowledge on whether there is the same risk for other statins, as well. The SLCO1B1 gene encodes for a liver-specific transporter protein. Currently, genetic screening is not cost-effective in this case (32).

In practice, for patients in whom one or more of the above effects mentioned have been identified, it is useful to measure the CK level before initiating statin ther-

TABLE 2. Statin-induced myopathy and risk factors for its appearance. The table below analyses both of the risk factor categories that could lead to developing statin-induced myopathy.

\begin{tabular}{|c|c|}
\hline Statin-dependent risk factors & Patient-dependent risk factors \\
\hline $\begin{array}{l}\text { - Relate to the type and dose of statin administered } \\
\text { - In vitro and in vivo studies have shown that lipophilic } \\
\text { statins (simvastatin, atorvastatin, lovastatin) cause } \\
\text { muscular symptoms more often than hydrophilic statins } \\
\text { (rosuvastatin, pravastatin, fluvastatin) } \\
\text { - High doses elicit muscular symptoms more often than } \\
\text { low doses } \\
\text { - For instance, for } 40 \mathrm{mg} \text { simvastatin administered, } \\
1 / 10,000 \text { cases of statin-induced myopathy were } \\
\text { reported, while the dose of } 80 \text { mg induced a } 10 x \text { growth } \\
\text { in cases: } 1 / 1,000 \text { cases }(20,28,29 \text { ) }\end{array}$ & $\begin{array}{l}\text { - Related to age (old age), gender (females), } \mathrm{BMI}\left(<11 \mathrm{~kg} / \mathrm{m}^{2}\right) \\
\text { - Pre-existing co-morbidities contribute to muscular side effects } \\
\text { and these include: } \\
\text { 1. Untreated hypothyroid, type } 1 \text { and type } 2 \text { diabetes mellitus, } \\
\text { renal and hepatic disorders, arterial hypertension } \\
\text { 2. High alcohol consumption, cocaine use, strenuous and } \\
\text { sustained physical exercise and simultaneous infections also } \\
\text { favour statin-induced myopathy }(1,19)\end{array}$ \\
\hline
\end{tabular}


apy, followed by periodic monitoring, clinical (regarding the debut of muscular symptoms) and serological (CK level) (1). In patients who do not exhibit any risk factors, no further investigations are necessary prior to statin treatment.

Through daily medical practice, there is a sense of "fear" regarding the possible side effects of the statin therapy and "unpleasant expectations" regarding the treatment and the development of unpleasant symptoms speeds up the - often self-directed - cessation of the therapy. Potential causes of myopathy and/or enzymatic increase without statin administration will be individually analysed and ultimately excluded. As shown before, in the case of a very high increase in CK ( $>50 x$ ULN, very rare), as in rhabdomyolysis and/or autoimmune myopathy, the patient is hospitalised immediately for specific treatment and monitoring.

In the literature, the recommendation to stop the statin therapy varies, but, generally, the therapy is stopped if muscular symptoms occur and are accompanied by high CK levels; in the case of identification on the initial screening of a CK elevation < 10x ULN, without muscular symptoms related to statin treatment, the statin will not be stopped, but the dosage will be reduced for safety reasons. CK level will be monitored for 2-6 weeks (1).

The European Society of Cardiology/European Atherosclerosis Society 2019 guideline mentions that, in 2-4 weeks, the statin is purified, so it could be assumed that symptoms that overrun this period of time are not linked to statin, so consequently the therapy can be resumed using the same statin (1). In the case of symptoms improvement and normalization of $\mathrm{CK}$, it is recommended that another statin is administered (if the initial statin is lipophilic, then the treatment would be resumed with a hydrophilic statin) in a low dose. There are studies showing that switching statins is a useful therapy strategy, usually better tolerated $(19,33)$. Monitoring the patients is highly necessary - if the CK levels are $>4 x$ ULN before treatment, starting therapy will be postponed and enzymes rechecked; routine monitoring is not necessary unless the patient develops symptoms or has risk factors, as mentioned before. If the symptoms return, a low dose of a third statin will be administered. The guideline now approves the use of alternative therapeutic schemes, once every two days or twice a week. This therapeutic approach requires patience, perseverance and represents a challenge for both clinician and patient, aiming to decrease the level of LDL-C to its target value, to reduce cardiovascular mortality. If the patient does not tolerate the new treatment scheme, then drug combination should be considered: statin + the lowest dose of ezetimibe, sequestrants of biliary acids or the more recent drug class of monoclonal antibodies: anti proprotein conver- tase subtilisin/kexin type 9 (PCSK9) inhibitors. The phase 3 clinical trials results prove that the PCSK9 inhibitors decrease the cardiovascular events post-lowering the LDL-C when added to statin and/or ezetimibe. There are no clinical studies data to assess their efficacy in monotherapy yet. Considering the high cost of this therapeutic approach, this statin + inhibitor combination therapy will be used in patients at high risk of atherosclerotic cardiovascular disease (1,34-36).

Regarding the treatment of common forms of myopathy, with enzymatic increases $<10 x U L N$, the muscle pain usually disappears when statin administration is stopped $(37,38)$, sometimes requiring analgesics. In the literature, there are discussions about a possible benefit of coenzyme Q10 supplementation. Statins block the production of ubiquinone/coenzyme Q10, reducing its circulation, which negatively feeds back on the energy producing function of the mitochondria in the skeletal muscle. This physiopathological links are hypothesised as a possible source of statin-induced myopathy, as mentioned before. The coenzyme Q10 supplementation increases its flow, but the results of multiple studies are contradictory: according to some, the simultaneous administration of coenzyme Q10 and statin reduces the statin-induced myopathy incidence $(39,40)$, while different coenzyme Q10 doses administered compared to placebo did not match the initial expectations, in practice, and did not influence the appearance or intensity of the muscular symptoms $(41,42)$.

On the same note, a recent analysis from 2020 did not demonstrate the efficacy of coenzyme Q10 supplementation in statin-induced myopathy patients, nor an improvement to the overall statin treatment scheme adherence (43).

\section{CONCLUSIONS}

The clinician will evaluate the cardiovascular risk of the patient and will establish the requirement of statin therapy, while appreciating any risks of developing statin-induced myopathy, the significant comorbidities such as hypothyroidism, chronic treatments and other drug-drug interactions that may occur.

If a risk of developing statin-induced myopathy arises, the level of CK is highly demanded; other disorders which elicit enzymatic increases will be excluded.

During the treatment scheme, if muscular problems arise, depending on the CK level, a patient-specific decision will be taken, considering the benefits and the risk of cessation the cholesterol-lowering therapy.

Rhabdomyolysis and the statin-induced autoimmune myopathy are severe, but rare complications; an early diagnosis enables an efficient therapeutic approach. In the long run, a new class of hypocholester- 
olemiant drugs could replace statins in patients at high risk of developing cardiovascular diseases.

Statistically, the more patients receive statin-based therapy, the higher the number of patients reporting muscular side effects, which can be proven by the interest of scientific guidelines and publications on this matter, providing recommendations regarding classification, monitoring and treatment approaches.

Conflict of interest: none declared Financial support: none declared

\section{REFERENCES}

1. 2019 ESC/AES Guidelines for the Management of the Dyslipidemia. European Heart Journal 2020;41(1):111-188.

2. Silverman MG, Ference BA, Im K, et al. Association between lowering LDL-C and cardiovascular risk reduction among different therapeutic interventions: a systematic review and meta - analysis. JAMA. 2016;316:1289-1297.

3. Ference BA, Graham I, Tokgozolu L, Catapano AL. Impact of lipids on cardiovascular health. JACC Health Promotion Series. J Am Cardiol. 2018;72:1141-1156.

4. Nawarskas JJ. HMG-CoA reductase inhibitors and coenzyme Q10. Cardiol Rev. 2005;13(2):76-9.

5. Tisha R. Joy, Robert A. Hegele, Narrative review: statin-related myopathy. Ann Intern Med. 2009;150:858-868.

6. Mammen AL. Statin-associated autoimmune myopathy. N Engl J Med. 2016;374(7):664669.

7. Nikolic D, Banach M, Chianetta R, et al. An overview of statin-induced myopathy and perspectives for the future. Expert Opin Drug Saf. 2020;19(5):601-615.

8. Cholesterol Treatment Trialists' (CTT) Collaborators, Mihaylova B, Emberson J, Blackwell L, Keech A, Simes J, Barnes EH, Voysey M, Gray A, Collins R, Baigent C. The effects of lowering LDL cholesterol with statin therapy in people at low risk of vascular disease: meta-analysis of individual data from 27 randomised trials. Lancet. 2012 Aug 11;380(9841):581-90.

9. Arnett DK, Blumenthal RS, Albert MA, et al. 2019 ACC/AHA Guideline on the Primary Prevention of Cardiovascular Disease: A Report of the American College of Cardiology/American Heart Association Task Force on Clinical Practice Guidelines. Circulation. 2019;140:e596-e646.

10. Sivakumar S, Lecky B. Statin-induced myopathy. BMJ. 2008;337:1159-62.

11. Sivashanmugarajah A, Fulcher J, Sullivan D, et al. Suggested clinical approach for the diagnosis and management of statin intolerance with an emphasis on musclerelated side-effects. Intern Med J. 2019;49(9):1081-1091.

12. Harper CR, Jacobson TA. Evidence-based management of statin-myopathy. Curr Atheroscler Rep. 2010:12(5):322-330.

13. Abd TT, Jacobson TA. Statin-induced myopathy: a review and update. Expert Opin Drug Saf. 2011;10(3):373-387.

14. Marcoff $L$, Thompson PD. The role of coenzymeQ10 in statin-associated myopathy: a systematic review. J Am Coll Cardiology. 2007;49(23):2231-7.

15. Young J, Florkowski C, Molyneux S, et al. Effect of coenzyme Q10 supplementation on simvastatin-induced myalgia. Am J Cardiol. 2007;100(9):1400-3.

16. Morandi L, Angelini C, Prelle A, et al. High plasma creatine kinase: review of the literature and proposal for a diagnostic algorithm. Neurol Sci. 2006;27(5):303-11.

17. Kley RA, Tobias Schimdt-Wilcke, Matthias Vorgerd. Differential diagnosis of hyperCKemia. Neurology International Open 2018;2:E72-E83.

18. Moghadam-Kia S, Oddis CV, Aggarwal R. Approach to asymptomatic creatine kinase elevation. Cleve Clin J Med. 2016;83(1):37-42.

19. Beckes JM, Ruisinger JF, Gibson CA, et al. Statin-associated muscle symptomsmanaging the highly intolerant. J Clin Lipidol. 2017;11(1):24-33.

20. Bruckert E, Hayem G. Mild to moderate muscular symptoms with high-dosage statin therapy in hyperlipidemic patients-the PRIMO study. Cardiovasc Drugs Ther. 2005;19(6):403-14.

21. Bosch X, Poch E, Grau J. Rhabdomyolysis and acute kidney injury. N Engl $J$ Med. 2009;361(1):62-72.

22. Thompson PD, Panza G, Zaleski A, Taylor B. Statin-associated side effects. J Am Coll Cardiol. 2016;67(20):2395-2410.

23. Selva-O'Callaghan A, Alvarado-Cardenas M, lago Pinal $F$, et al. Statin-induced myalgia and myositis: an update on pathogenesis and clinical recommendations. Expert Rev Clin Immunol. 2018:14(3):215-224.

24. Turner RM, Pirmohamed M. Statin-related myotoxicity: a comprehensive review of pharmacokinetic, pharmacogenomic and muscle components. J Clin Med. 2019;9(1):22.

25. Cristopher-Stine L, Basharat P. Statinassociated immune-mediated myopathy: biology and clinical implications. Current Opinion in Lipidology. 2017;28(2):186-192.

26. Hamann PD, Cooper RG, McHugh NJ, Chinoy H. Statin-induced necrotising myositis - a discrete autoimmune entity within the statin-induced myopathy spectrum. Autoimmunity Rev. 2013;12(12):1177-81.

27. Irvine NJ. Anti-HMGCR Myopathy: A Rare and Serious Side Effect of Statins. The Journal of the American Board of Family Medicine. 2020;33(5):785-788.

28. Davidson MH, Robinson JG. Safety of aggressive lipid management. Am Coll Cardiol. 2007;49(17):1753-62.
29. Collins R, Reith C, Emberson J, et al. Interpretation of the evidence for the efficacy and safety of statin therapy. Lancet. 2016;388(10059):2532-2561.

30. Sewright KA, Clarkson PM. Statin myopathy: incidence risk factors and pathophysiology. Curr Atheroscler Rep. 2007;9(5):389-96.

31. Chatzizisis YS, Koskinas KC, Misirli G, et al. Risk factors and drug interactions predisposing to statin-induced myopathy. Drug Saf. 2010;33:171-187.

32. Carr DF, O'Meara H, Jorgensen AL, et al. SLC01B1 genetic variant associated with statin-induced myopathy: a proof-of-concept study using the clinical practice research datalink. Clin Pharmacol Ther. 2013;94(6):695-701.

33. Pasternak RC, Smith SC, Bairey-Mertz CN, et al. ACC/AHA/NHLBI clinical advisory on the use and safety of statins. Stroke. 2002;33(9): 2337-41.

34. Chaudhary R, Garg J, Shah N, Sumner A. PCSK9 inhibitors: a new era of lipid lowering therapy. World J Cardiol. 2017;9(2):76-91.

35. Dallart RPF. PCSK 9 inhibition to reduce cardiovascular events. N Engl J Med. 2017;376(18):1790-1791.

36. Crismaru I, Pantea Stoian A, Bratu OG, et al. Low-density lipoprotein cholesterol lowering treatment: the current approach. Lipids in Health and Disease 2020;19(1):85.

37. Horodinschi R, Stanescu AMA, Bratu OG, et al. Treatment with statins in elderly patients. Medicina. 2019;55(11):721.

38. Gaman MA, Cozma MA, Dobrica EC, et al. Dyslipidemia: a trigger for coronary heart disease in Romanian patients with diabetes. Metabolites 2020;10(5):195.

39. Qu H, Guo M, Chai H, et al. Effects of coenzyme Q10 on statin-induced myopathy: an update meta-analysis of randomized controlled trials. Journal of the AHA. 2018;7(19).

40. Know L. Les mitochondries au coeur de la medicine du future. Dangles, 2019:151-163.

41. Bookstaver DA, Barkhalter NA, Hatzigeorgiou C. Effect of coenzyme Q10 supplementation on statin-induced myopathy. Am J Cardiol. 2012;110:526-529.

42. Ramachandran R, Wierbicki AS. Statins, muscle disease and mitochondria. J Clin Med. 2017 6(8):75.

43. Kennedy C, Koller Y, Surkova E. Effect of coenzyme Q10 on statin-associated myalgia and adherence to statin therapy. A systematic review and meta-analysis. Atherosclerosis. 2020;299:1-8. 